\title{
Inequities in Employment by Race, Ethnicity, and Sector During COVID-19
}

\author{
Jordan Gemelas ${ }^{1,2}$ (D) Jenna Davison ${ }^{1,2} \cdot$ Case Keltner $^{1} \cdot$ Samantha Ing $^{3}$
}

Received: 14 September 2020 / Revised: 5 January 2021 / Accepted: 7 January 2021 / Published online: 15 January 2021

(C) W. Montague Cobb-NMA Health Institute 2021

\begin{abstract}
Objective To determine whether people of Color experienced disparate levels of employment loss in frontline versus nonfrontline occupations during the onset of the COVID-19 pandemic.

Methods The Bureau of Labor Statistics Current Population Survey data was analyzed in a cross-sectional study. Percent change in number employed was tabulated quarterly for groups by race and ethnicity (Black or African American, Asian American, or Hispanic or Latinx compared to White or non-Hispanic or Latinx) and frontline occupation status between January 1 and June 30, 2020. Two-tailed two-sample tests of proportions were used to compare groups statistically.

Results More dramatic declines in number employed occurred in the Black or African American, Asian American, and Hispanic or Latinx groups. When stratified by sector, greater declines were noted in the Hispanic or Latinx and Asian American frontline, and Black or African American non-frontline groups when compared to the referent groups.

Conclusions Structural racism has further affected people of Color through differential employment loss during the onset of the pandemic, both overall and by sector. However, the effect of sector varies dramatically across racial and ethnic groups.

Policy Implications Because employment is an important social determinant of health and a potential risk factor for contracting COVID-19, these trends may provide important context for the prioritization of PPE and immunizations, as well as the provision of stable health insurance and income support for vulnerable workers.
\end{abstract}

Keywords Health disparities · Social determinants of health · Social vulnerabilities · COVID-19 · Occupational health

\section{Background}

In the context of recent economic downturn during the COVID-19 pandemic, employment may be a key social determinant of health contributing to racial and ethnic health disparities. Concerns have been raised about structural racism differentially impacting various groups as job opportunities become increasingly sparse [1-4].

The relationship between structural racism and employment prior to the COVID-19 pandemic has been established

Jordan Gemelas

gemelas@ohsu.edu

1 Oregon Health and Science University (OHSU) School of Medicine, Portland, OR, USA

2 OHSU-Portland State University School of Public Health, Portland, OR, USA

3 Highland Hospital Department of Internal Medicine, Oakland, CA, USA
[3]. Differential access to equal pay, paid sick leave, and unemployment benefits disproportionately harms people of Color [5]. Historically, Black, Hispanic and Latinx, and Asian American people have experienced rampant discrimination in the context of hiring and employment [6-9]. Population health and employment are also closely linked, as employment is a critical avenue to many social determinants of health [10]. Such determinants include health insurance, income, housing, and several other resilience factors, though the ability of workers to access these benefits varies widely across occupations [11-13]. Notably, employment is associated with improved physical and mental health, and employment frequently precedes health insurance coverage [14-16]. These ideas are particularly relevant given that the pandemic has worsened physical health and mental health outcomes across the board $[15,17,18]$.

However, employment now may be a risk factor for contracting COVID-19 for workers in certain occupations [2]. Compounding these issues are concerns that people of Color are more likely to work in positions that cannot be performed remotely, thereby limiting their protection against 
COVID-19 [2]. Thus, employment status and race and ethnicity can be further investigated with regard to occupation type and potential for exposure to the virus. Specifically, frontline workers in occupations responsible for performing critical inperson functions, such as healthcare delivery, food preparation, or public transportation (Table 1), may have limited teleworking options.

Many prior studies have confirmed that communities of Color comprised a disproportionate share of the baseline frontline workforce prior to the pandemic [19]. A recent review of 2019 data revealed that Black workers were more likely employed in essential industries and occupations with frequent exposure to infections, including the animal slaughtering and processing industry - a key source of isolated outbreaks during the pandemic - and the healthcare and social assistance industries [19]. Protections for American workers have also left these communities behind. The Families First Coronavirus Act provided federally mandated paid sick leave for those unable to work due to the impacts of COVID-19 and associated social distancing laws. However, people of Color account for $39 \%$ of workers affected by exemptions in the law [20].

While some policy analysis has been completed, few studies have investigated employment disparities that may have deepened in the wake of COVID-19 among frontline versus non-frontline workers across race and ethnicity in the United States $[2,4]$.

\section{Purpose}

In an attempt to understand the degree to which employment is a contributor to health disparities in communities of Color, the purpose of this analysis is to evaluate the association between race and ethnicity and (1) co-occurrence of job loss and (2) the likelihood of continued employment in a higher-risk frontline occupation following the onset of the COVID-19 pandemic.

\section{Methods}

We used the Bureau of Labor Statistics (BLS) Current Population Survey (CPS), stratified by race/ethnicity and employment sector. The CPS is administered monthly to a representative sample of 60,000 US households [21, 22]. Respondents report both racial identity and ethnic identity. Citizenship status is not ascertained. IRB approval was not necessary for use of public data.

First quarter (Q1) included January through March; second quarter (Q2) included April through June, 2020. In the US, restrictions and closures predominantly affected Q2; Q1 was used as the functional baseline. While some seasonal variation may exist, we were concerned about the effect of year-to-year

Table 1 Frontline and non-frontline designation by BLS CPS category, number employed Q1 and Q2 2020

\begin{tabular}{llll}
\hline & Q1 employed & Q2 employed & Change (\%) \\
\hline Frontline occupations & & & $-1,504,342(-6.47 \%)$ \\
Healthcare practitioners and support & $23,258,585$ & $21,754,243$ & $-9222(-0.45 \%)$ \\
Protective service & $2,033,623$ & $2,024,401$ & $-3,693,883(-23.93 \%)$ \\
Food preparation, serving related, postal service, and transportation & $15,433,970$ & $11,740,087$ & $-414,959(-27.04 \%)$ \\
Building and grounds cleaning, maintenance, and personal care and service & $1,534,838$ & $1,119,879$ & $+113,791(+6.51 \%)$ \\
Farming, mining, fishing, and forestry & $1,747,891$ & $1,861,682$ & $+72,082(+0.98 \%)$ \\
Construction and extraction & $7,374,725$ & $7,446,807$ & $-86,504(-6.46 \%)$ \\
Installation, maintenance, and repair & $1,339,489$ & $1,252,985$ & $-702,601(-5.52 \%)$ \\
Production & $12,721,088$ & $12,018,487$ & $-73,740(-0.97 \%)$ \\
Non-frontline occupations & & $-55,198(-2.47 \%)$ \\
Management, business, and financial & $7,636,314$ & $7,562,574$ & $-23,073(-1.46 \%)$ \\
Computer and mathematical & $2,232,121$ & $2,176,923$ & $+35,963(+1.95 \%)$ \\
Architecture and engineering & $1,583,695$ & $1,560,622$ & $-28,974(-2.51 \%)$ \\
Life, physical, community, and social services & $1,848,471$ & $1,884,434$ & $-1,628,215(-12.46 \%)$ \\
Legal & $1,153,815$ & $1,124,841$ & $-1,140,055(-18.57 \%)$ \\
Education, training, and library & $13,068,996$ & $11,440,781$ & $-3,009,524(-6.25 \%)$ \\
Arts, design, entertainment, sports, and media & $6,137,785$ & $4,997,730$ & $45,147,878$ \\
Business, sales, office employment, and other & $48,157,402$ & & \\
\hline
\end{tabular}

Employment numbers are based on the North American Industry Classification System (NAICS) quarterly census of available data from private and government employment and wages in all counties within the US Bureau of Labor Statistics (BLS). Q1 employment is based on numbers from January, and Q2 employment is based on numbers from June 
variation as well in deciding against using Q2 2019 as a baseline interval. Additionally, the changes from Q1 to Q2 in 2019 were relatively minimal compared to similar changes in 2020 (Fig. 1). We assigned frontline designation (defined as occupation categories that are typically considered critical function and that require in-person interaction) for workers based on reported CPS categories. Table 1 details descriptive trends among each occupation category over the study interval for all private and government employment [23].

We examined percent change in number employed between groups based on racial (White, Asian American, or Black or African American) and ethnic identities (Hispanic or Latinx or non-Hispanic or Latinx) from Q1 to Q2 in 2020. We used White and non-Hispanic or Latinx as the referent groups. We then carried out a planned stratification by frontline occupation status. Frontline groups were compared to frontline groups, and a similar scheme was used for non-frontline groups. Each group was compared statistically to the referent categories using a two-sample, two-tailed test of proportions without continuity correction. The result of the two-sample test of proportions indicated whether a group demonstrated a statistically different percent change in number employed, and the magnitude and direction of such change. Due to the multiple comparisons required to execute this analysis, a reduced alpha threshold was used per Bonferroni familywise error rate correction to adjust for the increased likelihood of type 1 error (alpha $=0.0083$, confidence level $=99.17 \%$ ). . Computations were performed in $R$, an open-source statistical analysis tool.

\section{Results}

Overall, the number of employed workers decreased by $12.0 \%$ from Q1 to Q2 2020, while the number of employed increased by $0.9 \%$ from Q1 to Q2 in 2019. With regard to frontline positions, the number employed decreased by $17.2 \%$ from Q1 to Q2 2020, while it increased by $2.9 \%$ from Q1 to Q2 in 2019. For non-frontline positions, the number employed decreased by $7.9 \%$ from Q1 to Q2 in 2020, while it decreased 0.7\% from Q1 to Q2 in 2019. Descriptive trends by race or ethnicity can be found in Fig. 1 and Tables 2 and 3 .

Each racial or ethnic group exhibited dramatic decreases in number employed, with each group demonstrating statistically significant differences in magnitude of loss (Asian American $-15.14 \%$; Black or African American - 13.95\%; White $11.36 \%$; Hispanic or Latinx $-15.68 \%$; non-Hispanic or Latinx $-11.24 \%$ ) (Table 3).

Relative to the referent analytic groups, the Asian American and Hispanic or Latinx non-frontline groups demonstrated a modest excess decrease (Asian American nonfrontline $-7.86 \%$; difference $-0.42 \%\{-0.39 \%,-0.45 \%\}$ ) or lesser decrease (Hispanic or Latinx non-frontline $-6.21 \%$; difference $+1.97 \%\{+1.95 \%,+1.99 \%\})$. In contrast, the Black or African American non-frontline group exhibited a much larger percent decrease relative to the referent group $(-12.26 \%$; difference $-4.81 \%\{-4.78 \%,-4.84 \%\})$, suggesting marked heterogeneity in impact between racial and ethnic groups. Complete results are found in Table 3.

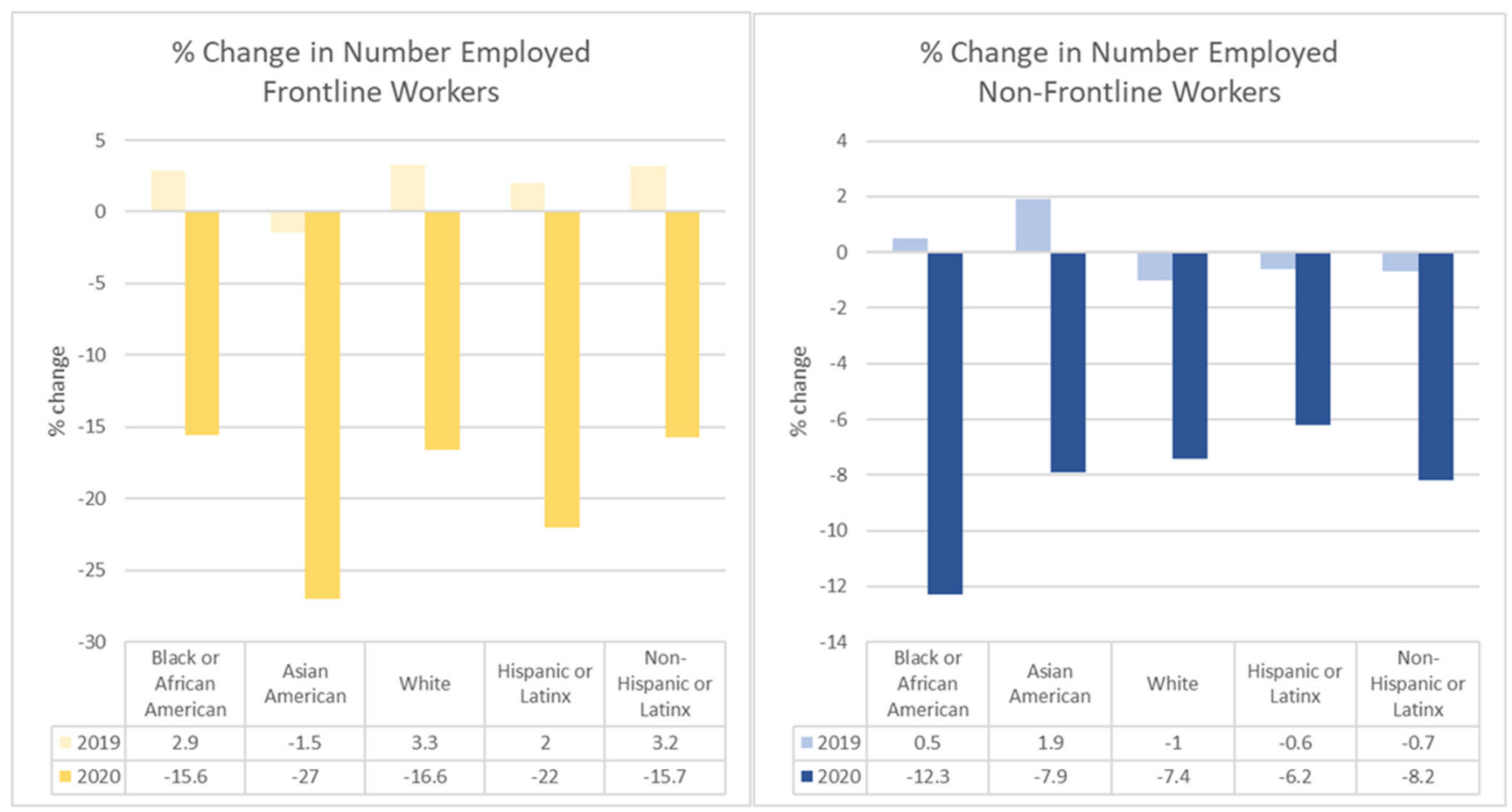

Fig. 1 Percent change in number employed, Q1 to Q2, 2019 and 2020, by racial or ethnic identity and frontline occupation status 


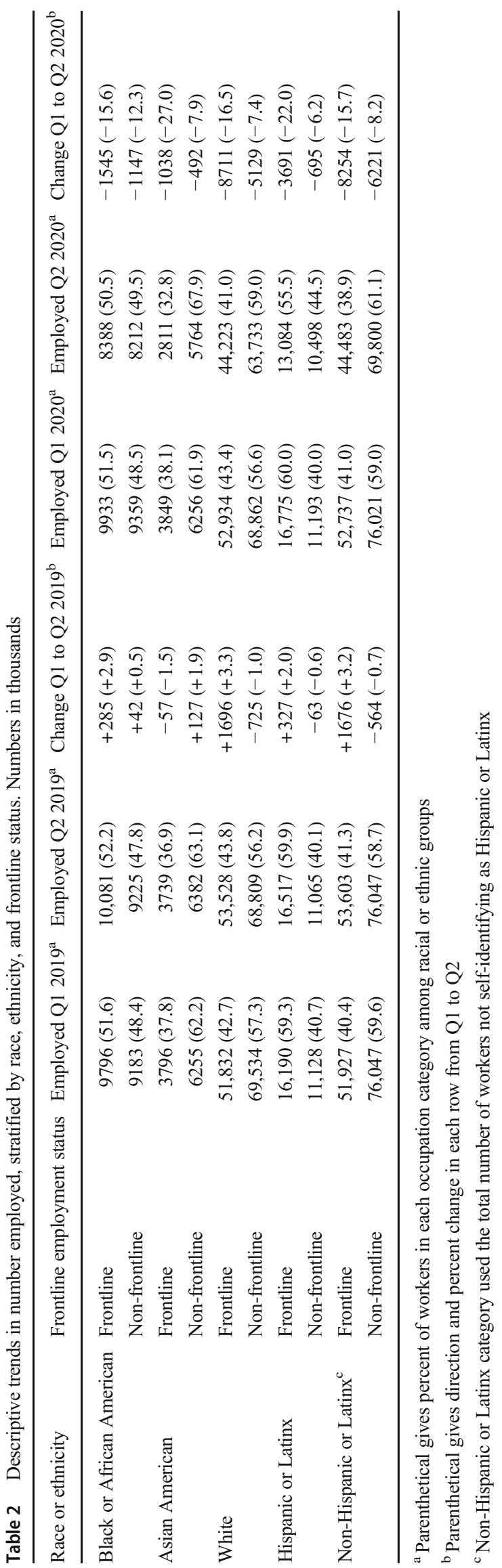

\section{Discussion}

This analysis demonstrates that previously employed workers in marginalized populations exhibited greater likelihood of job loss during the recent economic decline attributable to COVID-19.

Upon performing the planned stratification, it was further discovered that Black or African American workers were much more likely to lose or leave lower-risk and potentially more secure non-frontline positions relative to White workers in the first half of 2020. By contrast, we found that the disparity in the decreases in number employed among Hispanic or Latinx and Asian American workers was driven to a somewhat greater extent by frontline positions. For Hispanic or Latinx workers, we suggest this could be in part due to a significantly greater proportion of Hispanic or Latinx workers being employed in frontline positions at baseline $(60.0 \%)$ relative to other groups (41.0\%), which could have distorted our results to some extent. Additionally, we speculate that migrant workers with Hispanic or Latinx identity could be more likely to be insecurely employed in frontline jobs. However, Asian American workers (38.1\%) were not employed in frontline positions at a greater proportion compared to other groups. During the pandemic, Asian American workers experienced a dramatic increase in discrimination and interpersonal racism, which could partially explain this trend if this translated to a significant efflux of Asian American workers in the job market (on voluntary or involuntary grounds) [17, 24]. The distribution of specific frontline occupations among a racial or ethnic group may also differ. For example, a group with more food service workers may have less secure employment, while a group with a higher proportion of physicians may have more secure employment. Furthermore, we would predict that the frequency of workers voluntarily leaving jobs (versus being laid off) may have differed between groups [2]. Workers with less robust financial reserves might feel no option but to maintain a high-risk job despite a desire to isolate for self-protection. Baseline financial resources could in turn be tied to racial or ethnic identity due to historical structural oppression and privilege [2].

Though our results confirm that COVID-19 has accentuated employment inequalities in the USA, larger issues with employment disparities have persisted for decades [5]. Structural racism from (1) the normalization of Black or African American workers being employed at a higher proportion in at-risk occupations to (2) the use of racism against Asian Americans and Hispanic or Latinx people as a political tool may be driving these disparities now [25]. These issues are not new - they have simply been brought into acute focus by the pandemic $[1,19,25]$. In combination with increased publicity of incidents involving police use of force, significant disparities in COVID-19 incidence and outcomes, and explicit discriminatory narratives used by political figures, a long overdue increased awareness of racism as a public health emergency has emerged in the mainstream national consciousness $[25,26]$. 
Table 3 Analytic results, comparison of percent decrease in employment, stratified by race/ethnicity, and frontline status

\begin{tabular}{|c|c|c|c|c|}
\hline Race or ethnicity & Frontline employment status & Crude percent decrease $(99.0 \% \mathrm{CI})$ & $\begin{array}{l}\text { Stratified percent } \\
\text { decrease }(99.5 \% \mathrm{CI})\end{array}$ & $\begin{array}{l}\text { Difference in percent change } \\
\text { compared to referent category }\end{array}$ \\
\hline \multirow[t]{2}{*}{ Black or African American } & Frontline & $13.95(13.93,13.97)$ & $15.55(15.52,15.59)$ & $-0.90(+0.87,+0.94)$ \\
\hline & Non-frontline & & $12.26(12.23,12.29)$ & $-4.81(-4.78,-4.84)$ \\
\hline \multirow[t]{2}{*}{ Asian American } & Frontline & $15.14(15.11,15.17)$ & $26.97(26.90,27.03)$ & $-10.51(-10.45,-10.57)$ \\
\hline & Non-frontline & & $7.86(7.83,7.89)$ & $-0.42(-0.39,-0.45)$ \\
\hline \multirow[t]{2}{*}{ White } & Frontline & $11.36(11.36,11.37)$ & $16.46(16.44,16.47)$ & $\operatorname{Ref}^{f}$ \\
\hline & Non-frontline & & $7.45(7.44,7.46)$ & Ref \\
\hline \multirow[t]{2}{*}{ Hispanic or Latinx } & Frontline & $15.68(15.66,15.70)$ & $22.00(21.97,22.03)$ & $-6.35(-6.32,-6.38)$ \\
\hline & Non-frontline & & $6.21(6.19,6.23)$ & $+1.97(+1.95,+1.99)$ \\
\hline \multirow[t]{2}{*}{ Non-Hispanic or Latinx $\mathrm{x}^{\mathrm{e}}$} & Frontline & $11.24(11.23,11.25)$ & $15.65(15.64,15.67)$ & Ref \\
\hline & Non-frontline & & $8.18(8.17,8.19)$ & Ref \\
\hline
\end{tabular}

${ }^{\mathrm{d}}$ Confidence level $99.17 \%$ due to multiple comparisons

${ }^{\mathrm{e}}$ Non-Hispanic or Latinx category used the total number of workers not self-identifying as Hispanic of Latinx

${ }^{\mathrm{f}}$ Referent group for computations. For racial identities, White was used as the referent category. For ethnic groups, non-Hispanic or Latinx was used as the referent category. For occupation type, frontline groups were compared to referent frontline groups; an identical scheme was used for non-frontline groups

\section{Strengths and Limitations}

To our knowledge, this one of the few analyses to investigate employment changes by race, ethnicity, and employment sector using nationally representative retrospective data during the COVID-19 pandemic. While the broad categories used here neglect marked within-group variability and rely on the social construct of race, they reflect exposure to structural racism in the setting of American society [27]. Importantly, race is a contextual characteristic that varies in meaning across space and time, and has social, not biological, significance [27].

Our study has several limitations, most of which can be attributed to the original dataset. Variables such as length of time in occupation, wages, specific geographic information, and more granular breakdown of career types was not available and could have been useful additions. Additionally, the referent categories (non-Hispanic or Latinx and White) include many subpopulations that are indeed subject to racism and other forms of oppression. Smaller racial and ethnic groups were not reported, and further nuance could be added by evaluating the effect of COVID-19 on the employment trends in these groups. Future analyses with primary data may be able to evaluate these factors at the expense of having a nationally representative sample. Additionally, while the break point between Q1 and Q2 closely approximated most of the impacts of COVID-19 on the job market, some effects preceded April 1, 2020. If job losses occurred significantly prior to April 1, we predict it would have biased many of the observed effects in the direction of the null.

\section{Public Health Implications}

The onset of the COVID-19 pandemic has accentuated employment disparities. This study improves understanding of the associations of race and ethnicity and social conditions given COVID19 health disparities and may guide policy decision making. However, the evidence presented here is relevant only in the context of explicit prioritization of health equity in policy design.

People of Color were subject to overall increased risk of job loss during the initial phases of COVID-19, likely as a product of structural and interpersonal racism. These job losses were considerable compared to 2019 trends. Black or African American workers were more likely to lose or leave non-frontline positions, and Hispanic or Latinx and Asian American workers were more likely to experience job loss in frontline positions relative to the referent groups. Because employment is both an important social determinant of health, and, for frontline workers, a potential risk factor for exposure to COVID-19, these trends may provide a partial mechanistic understanding of the striking health disparities previously identified (i.e., communities of Color at increased risk for infection, severe illness, and death).

To protect the health and safety of oppressed populations, policymakers must take immediate action to implement greater worker protections for communities of Color. Policies directed at improving access to stable health insurance, income support, COVID-19 immunizations, and PPE for frontline workers could reduce the differential health impacts of COVID-19 on people of Color. Future studies should examine the relative benefits of each of these interventions as the initial phases of vaccine distribution unfold. 
Data Availability See references [21-23].

\section{Compliance with Ethical Standards}

Conflict of Interest The authors declare that they have no conflicts of interest.

\section{References}

1. Coronavirus layoffs more severely threaten housing security for Asian, Latinx and Black Households. April 27, 2020. Accessed June 28, 2020. https://www.marketwatch.com/press-release/ coronavirus-layoffs-more-severely-threaten-housing-security-forasian-latinx-and-black-households-2020-04-27

2. Gould E, Wilson V. Black workers face two of the most lethal preexisting conditions for coronavirus- racism and economic inequality. Economic Policy Institute June 1, 2020. Accessed June 24, 2020. https://www.epi.org/publication/black-workerscovid/

3. Yearby R. Racial disparities in health status and access to healthcare: the continuation of inequality in the United States due to structural racism. Am J Econ Sociol. 2018;77:1113-52.

4. Montenovo L, Jiang X, Rojas FL, et al. Determinants of disparities in Covid-19 job losses. Natl Bur Econ Res; 2020:w27132. https:// doi.org/10.3386/w27132

5. Yearby R, Mohapatra S. Law, structural racism, and the COVID-19 pandemic. J Law Biosci. 2020;7. https://doi.org/10.1093/jlb/ lsaa036.

6. Quillian L, Pager D, Hexel O, Midtbøen AH. Meta-analysis of field experiments shows no change in racial discrimination in hiring over time. PNAS. 2017;114(41):10870-5.

7. Mcmurtry CL, Findling MG, Casey LS, et al. Discrimination in the United States: experiences of Asian Americans. Health Serv Res. 2019;54(S2):1419-30. https://doi.org/10.1111/1475-6773.13225.

8. Tessler H, Choi M, Kao G. The anxiety of being Asian American: hate crimes and negative biases during the COVID-19 pandemic. Am J Crim Justice. 2020;45(4):636-46. https://doi.org/10.1007/ s12103-020-09541-5.

9. Findling MG, Bleich SN, Casey LS, Blendon RJ, Benson JM, Sayde JM, et al. Discrimination in the United States: experiences of Latinos. Health Serv Res. 2019;54:1409-18. https://doi.org/10. 1111/1475-6773.13216

10. van der Noordt M, IJzelenberg H, Droomers M, Proper KI. Health effects of employment: a systematic review of prospective studies. Occup Environ Med. 2014;71(10):730-6. https://doi.org/10.1136/ oemed-2013-101891.

11. Forchuk C, Dickins K, Corring D. Social determinants of health: housing and income. hcq. 2016;18(SP):27-31. https://doi.org/10. 12927/hcq.2016.24479

12. Bor J, Cohen GH, Galea S. Population health in an era of rising income inequality: USA, 1980-2015. Lancet. 2017;389(10077): 1475-90. https://doi.org/10.1016/S0140-6736(17)30571-8.

13. McWilliams JM, Meara E, Zaslavsky AM, Ayanian JZ. Health of previously uninsured adults after acquiring Medicare coverage. JAMA. 2007;298(24):2886-94. https://doi.org/10.1001/jama.298. 24.2886
14. Caicedo M, van Gameren E. Desempleo y salud mental en la población de origen hispano en Estados Unidos: un análisis epidemiológico [Unemployment and mental health among Hispanics in the US: an epidemiological analysis]. Cien Saude Colet. 2016;21(3):955-66. Spanish. https://doi.org/10.1590/141381232015213.16592014 .

15. Tsamakis K, Triantafyllis AS, Tsiptsios D, Spartalis E, Mueller C, Tsamakis C, et al. COVID-19 related stress exacerbates common physical and mental pathologies and affects treatment. Exp Ther Med. 2020;20(1):159-62. https://doi.org/10.3892/etm.2020.8671.

16. Lipton BJ. Association between health insurance and health among adults with diabetes: evidence from Medicare. J Am Geriatr Soc. 2020;68(2):388-94. https://doi.org/10.1111/jgs.16238.

17. van Dorn A, Cooney RE, Sabin ML. COVID-19 exacerbating inequalities in the US. Lancet. 2020;395(10232):1243-4. https://doi. org/10.1016/S0140-6736(20)30893-X.

18. Zvolenksy MJ, Garey L, Rogers AH, et al. Psychological, addictive, and health behavior implications of the COVID-19 pandemic. Behav Res Ther. 2020;134:103715. https://doi.org/10.1016/j.brat. 2020.103715.

19. Hawkins D. Differential occupational risk for COVID-19 and other infection exposure according to race and ethnicity. Am J Ind Med. 2020;63:1-4. https://doi.org/10.1002/ajim.23145.

20. Long M, Rae M. Gaps in the emergency paid sick leave law for health care workers. Kaiser Family Foundation June 17, 2020. Accessed July 3, 2020. https://www.kff.org/coronavirus-covid-19/ issue-brief/gaps-in-emergency-paid-sick-leave-law-for-health-careworkers/

21. US Bureau of Labor Statistics. Labor Force Statistics from the Current Population Survey. E-13. Employed persons by sex, occupation, class of worker, full- or part-time status, and race. Published July 2, 2020. https://www.bls.gov/web/empsit/cpsee e13.htm

22. US Bureau of Labor Statistics. Labor Force Statistics from the Current Population Survey. E-14. Employed Hispanic or Latino workers by sex, occupation, class of worker, full- or part-time status, and detailed ethnic group. Published July 2, 2020. https://www. bls.gov/web/empsit/cpsee_e14.htm

23. US Bureau of Labor Statistics. North American Industry Classification System (NAICS) at Bureau of Labor Statistics (BLS). https://www.bls.gov/bls/naics.htm

24. Kantamneni N. The impact of the COVID-19 pandemic on marginalized populations in the United States: a research agenda. J Vocat Behav. 2020;119:103439. https://doi.org/10.1016/j.jvb.2020. 103439.

25. Devakumar D, Shannon G, Bhopal S, Abubakar I. Racism and discrimination in COVID-19 responses. Lancet. 2020;395(10231):1194. https://doi.org/10.1016/s0140-6736(20) 30792-3.

26. Fortuna L, Tolou-Shams M, Robles-Ramamurthy B, Porche M. Inequity and the disproportionate impact of COVID-19 on communities of color in the United States: the need for a trauma-informed social justice response. Psychol Trauma. 2020;12:443-5. https:// doi.org/10.1037/tra0000889.

27. Jones CP. Invited commentary: "race," racism, and the practice of epidemiology. Am J Epidemiol. 2001;154(4):299-304. https://doi. org/10.1093/aje/154.4.299.

Publisher's Note Springer Nature remains neutral with regard to jurisdictional claims in published maps and institutional affiliations. 